\title{
Quantum Geometry of Loops and the Exact Solubility of Non-Abelian Gauge Chern-Simons Theory - II
}

\author{
M. Awada* \\ Physics Department, Imperial College, London SW7, UK
}

\begin{abstract}
We quantize non-abelian Chern-Simons gauge theory in three dimensions in the presence of Wilson lines. We determine the theory dynamically in terms of the geometry of loops and show that it is exactly soluble. Remarkably the quantum loop equations are linear for $S^{3}$ and they possess a class of solutions, among which is a non-critical Fermi string theory. Using these solutions we determine various important identities relevant to knot theory discovered recently by E. Witten, in particular, we show that the loop equation yields precisely the full exact skein relation of knot theory. As a byproduct we show that the partition function of an unknotted Wilson loop on $S^{3}$ is nothing but the character of $S U(2)$ in which the rotations are $S U(N)$ valued fractional angles. Furthermore, we generalize our solutions to the case where the manifold $M_{3}$ is oriented, closed, and non-simply connected with $H_{1}\left(M_{3}\right)=0$ (a homology 3-sphere).
\end{abstract}

\section{Introduction}

It has been long conjectured that closed strings play the role of elementary excitations in the confining phase of a gauge theory $[1,2]$. This has led to various attempts to formulate Q.C.D. as a chiral theory in loop space $[3,4]$. The strong similarity between the field equations of chiral fields in $d=2$ space-time dimensions and the Yang-Mills field equations in loop space suggest that there exists an exact solution to the gluon dynamics. In fact, it has been suggested that in the $N=\infty$ limit, Q.C.D. is equivalent to a Fermionic string theory [5]. Unfortunately this scheme did not go very far due to various problems in the renormalization theory of fields on loop space. Nevertheless, the idea itself is very attractive and has potential applications in other types of non-abelian gauge theories. In this paper, we will formulate the Chern-Simons (CS) non-abelian gauge theory in three space-time dimensions in terms of quantum geometry of loops. We will show that the theory is exactly soluble in loop space, and is equivalent to a Fermionic string theory. Our approach will be an alternative new

* Address after $1^{\text {st }}$ October 1989: Physics Department, University of Florida, Gainsville, FL 32611, USA 
proof of the recent assertion that the CS theory in $d=3$ is an exactly soluble theory [6]. In fact, Witten has given a general mechanism for classifying knots on arbitrary 3-d manifolds and how to calculate them from a surgery presentation. The basic element in his construction is the quantum field theory of the CS theory. His method is based on canonical quantization, while ours will be based on the quantum loop representation of the CS theory. The solutions we will obtain shows that the partition function satisfies certain factorization identities which are nothing but the Witten's identities discovered in connection with knot theory [6]. The full technical presentation of this paper will be published elsewhere [7].

Our starting point is not to consider Yang-Mills action in $d=3$, but rather the topological Chern-Simons three forms

$$
I_{c \cdot s}=n / 4 \pi \cdot \int_{M_{3}} \operatorname{tr}(A \wedge d A+2 / 3 A \wedge A \wedge A),
$$

where the trace denotes an invariant bilinear form on the lie algebra $G$ which acts as a symmetry group of (1.1). The action (1.1) is invariant under the infinitesimal gauge transformations:

$$
\delta A=D \varepsilon=d \varepsilon+[A, \varepsilon],
$$

but not under the finite gauge transformations:

$$
A^{\prime}=U A U^{-1}+U d U^{-1},
$$

this is because the gauge group $J$ (which is the set of maps from $M_{3} \rightarrow G$ ) has disconnected components, and they are classified by $\pi_{0}(J) \simeq \pi_{3}(G) \simeq Z$ for compact simple groups $G$ and $M_{3}=S^{3}$. For an arbitrary 3-d manifold the $\pi(M, G)$ can be calculated to be the integers up to a finite piece which is the fundamental homotopy group of $M$. Under the actions of (1.3), Eq. (1.1) transforms as $I_{c \cdot s}+$ const $m$. This implies that we can make expiI $I_{c \cdot s}$ invariant under global transformations by choosing $n$ to be an integer; $n \in Z$. In fact, this is the quantization condition on the Planck's constant which appears as the parameter in the quantum theory of (1.1) [16]:

$$
W\left(M_{3}, 0\right)=\int D A \exp i I_{c \cdot s} .
$$

In addition to $I_{c \cdot s}$ we need another type of gauge invariant observable, and these are the Wilson lines which are non-local in the gauge connections $A$ :

$$
\Phi(C, R):=\operatorname{tr}_{R} P \exp \oint_{C} A,
$$

where the trace has been taken over the irreducible representation $R$ of $G ; P$ is the path ordering along the closed path $C$, and $A$ is the gauge one-form on $M_{3} . \Phi(C, R)$ is actually the trace of an element $\Psi(C)$ of the holonomy group. The basic properties of $\Psi(C)$ are:

i) Reparametrization invariance: Where $s \rightarrow f(s)$ is an arbitrary reparametrization transformation.

ii) Under the action of gauge groups (1.3), $\Psi(C)$ transforms as:

$$
\Psi\left(C_{x y}\right) \rightarrow U^{-1}(x) \Psi\left(C_{x y}\right) U(y),
$$

where $C_{x y}$ is the open path between two points $x$ and $y$. 
iii) Under infinitesimal variations of the path, we have

$$
\delta \Psi(C)=\int_{0}^{2 \pi} P\left(F_{\mu v}(x(s)) \exp \oint_{C} A\right) \dot{x}^{\nu}(s) \delta x^{\mu}(s) d s ; \quad \mu=1,2,3 .
$$

These properties, imply that $\Phi(C, R)$ is reparametrization and gauge invariant, and moreover a path independent quantity. The last property follows from the fact that for a Yang-Mills Chern-Simons theory the field equation is

$$
F=0 \text {. }
$$

However, the independency of the path is only true for an unknotted loop on a simply connected manifold and for $d=3$ this is $S^{3}$. For non-simply connected 3-d manifolds the path dependence of $\Phi(C, R)$ is classified by the homomorphism $h$ from $\pi_{1}(M) \rightarrow G$, where $\pi_{1}(M)$ is the fundamental homotopy group of $M_{3}$. On the other hand, if the loop is knotted, even on $S^{3}$, Eq. (1.6) will break down and this is to do with the existence of extra topological information associated with the embedding of $C$ in $M_{3}$ (cf. Sect. 4 for details). Therefore, $\Phi(C, R)$ looks to be a very good candidate for a quantum observable in the theory, and hence we would be interested to study the topological quantum field theory associated with $\Phi(C, R)$ :

$$
\left\langle\Phi\left(C_{1}, R_{1}\right) \ldots \Phi\left(C_{n}, R_{n}\right)\right\rangle=\int D A \exp i I_{c \cdot s} \cdot \prod_{i=1}^{n} \Phi\left(C_{i}, R_{i}\right)
$$

this ansatz has been studied recently by E. Witten, who showed that it can be used to classify all knot polynomials on $S^{3}$ and in general on $M$. In this paper we will calculate (1.8) starting from quantum loop geometry; and show how to obtain explicit solutions for:

$$
W\left(M_{3}, C, R\right):=\langle\Phi(C, R)\rangle,
$$

on loop space. In particular, we will determine $W$ in terms of the partition function of a Fermionic string theory, and prove that the corresponding expression satisfies certain factorization knot identities recently discovered in [6]. Furthermore, we will show that the linear loop equation yields the full exact skein relation of knot theory, in which the renormalized partition function plays the role of the knot polynomial.

\section{Quantum Loop Geometry}

Our strategy is to obtain the quantum field equation satisfied by $W(C)$ in loop space and then look for solutions for this equation. From Eqs. (1.9) and (1.6) it follows that

$$
\frac{\delta}{\delta x^{\mu}(s)} W\left(M_{3}, C, R\right)=\dot{x}^{\nu}(s)\left\langle\operatorname{tr}_{R} P\left(F_{\mu \nu}(x(s)) \exp \oint_{C} A\right)\right\rangle, \quad \dot{x}^{\mu}(s):=d x^{\mu}(s) / d s,
$$

therefore, classically the field equation is

$$
\frac{\delta}{\delta x^{\mu}(s)} W\left(M_{3}, C, R\right)=0,
$$


however, we would like to calculate (1.2) quantum mechanically, and this is the crucial step in this program. To do that we consider

$$
\langle\Psi(C)\rangle:=\int D A \operatorname{expiI_{c}\cdot s} \cdot P \exp \oint_{C} A
$$

Equation (2.3) is not gauge invariant since $\psi(C)$ is not. However, we can make a dummy change of variable $A \rightarrow A+\delta A$ in (2.3) and use

$$
\delta \psi(C)=\int_{0}^{2 \pi} d s\left(P \exp \int_{0}^{x} A_{\mu} d x^{\mu}\right) \delta A(x(s)) \cdot\left(P \exp \int_{x}^{2 \pi} A_{\mu} d x^{\mu}\right),
$$

to obtain the following identity:

$$
\left\{\frac{i n}{4 \pi}\left\langle\varepsilon^{\mu \varrho \sigma} \operatorname{tr} F_{\varrho \sigma}(x(z)) \psi(x, x)\right\rangle-\oint_{C_{x x}} d y^{\mu} \delta(x(z)-y)\left\langle\operatorname{tr} T^{a} \psi(x, y) T^{a} \psi(y, x)\right\rangle\right\}=0 .
$$

For $S U(N)$

$$
\operatorname{tr}\left(T_{i j}^{a} T_{k l}^{a}\right)=\left(\delta_{i k} \delta_{j l}-\frac{1}{N} \delta_{i j} \delta_{k l}\right)
$$

The dummy change of variable is equivalent to the statement that:

$$
\int D A \frac{\delta}{\delta A}\left(\exp i I_{c \cdot s} \cdot P \exp \oint_{C} A\right)=0,
$$

is a total divergence. The first term in (2.5) comes from varying the expi $I_{c \cdot s}$ in (2.3) and the second term follows from the variation of the Wilson loop term $\psi(C)$ using (2.4). The quantum fluctuation of $\langle F \cdot \psi(C)\rangle$ in the presence of the holonomy factor $\langle\psi(C)\rangle$ can now be calculated by requiring the cancellation of the two terms in (2.5) we finally obtain the following identity:

$$
\left\langle\operatorname{tr} F_{\mu \nu}(x(s)) \psi(x, x)\right\rangle=\frac{2 \pi i}{n} \oint_{C_{x x}} \varepsilon_{\mu \nu \varrho} \delta(x(s)-y)\left\{W^{2}\left(C_{x y}, C_{y x}\right)-\frac{1}{N} W(C)\right\} d y^{\varrho},
$$

inserting this into Eq. (2.1) we deduce the quantum loop equations satisfied by $W(C)$, namely:

$$
\frac{\delta}{\delta x^{\mu}(s)} W(C, R)=\frac{2 \pi i}{n} \oint_{C_{x x}} d y^{\varrho} \dot{x}^{\sigma}(s) \varepsilon_{\mu \sigma \varrho} \delta(x(s)-y)\left\{W^{2}\left(C_{x y}, C_{y x}\right)-\frac{1}{N} W(C)\right\},
$$

where $C_{x x}$ is the closed contour $C$ with the marked point $x$, and $W^{2}\left(C_{1}, C_{2}\right)$ is defined to be:

$$
W^{n}\left(C_{1}, R_{1}, \ldots, C_{n}, R_{n}\right)=\left\langle\Phi\left(C_{1}, R_{1}\right) \ldots \Phi\left(C_{n}, R_{n}\right)\right\rangle .
$$

If the loop $C$ is smooth i.e. $x(s) \neq y$ i.e. does not have a self intersection at the point $x(s)$, then the right-hand side of (2.7) vanishes because of the $\delta$-functions, otherwise the point $y$ splits the loop into two closed loops, $C_{1}$ and $C_{2}$ (cf. Fig. 1). For later 
purposes we will rewrite Eq. (2.7) in terms of area variations

$$
\begin{aligned}
\delta \sigma^{\varrho \sigma}: & =\frac{1}{2} \oint_{\delta C} x^{\varrho}(s) d x^{\sigma}, \\
\frac{\delta}{\delta \sigma^{\mu}}: & =\frac{1}{2} \varepsilon^{\mu \varrho \sigma} \frac{\delta}{\delta \sigma^{\varrho \sigma}}
\end{aligned}
$$

(where $\delta \sigma^{\mu v}$ is the area enclosed by the small deformations $\delta C$ ) which is related to the path variation $\frac{\delta}{\delta x^{\mu}(s)}$ by:

$$
\frac{\delta}{\delta x^{\mu}(s)}=\dot{x}_{v}(s) \frac{\delta}{\delta \sigma_{\mu \nu}(x(s))}+\sum_{i=1}^{m} \partial_{\mu}^{x_{i}} \delta\left(s-s_{i}\right) .
$$

After rescaling $W(C, R) \rightarrow \frac{1}{N} W(C, R)$ we have:

$$
\frac{\delta}{\delta \sigma^{\mu}(s)} W(C, R)=\frac{2 \pi i}{m N} \oint_{C_{x x}} d y^{\mu} \delta(x(s)-y)\left\{W^{2}\left(C_{1}, C_{2}\right)-W(C)\right\},
$$

where

$$
m:=n+C_{2}(G) / 2 ; \quad k=m N .
$$

In (2.10) we have replaced the integer $n$ by $k$. This requirement has been explained fully in [6]. Briefly, it comes about upon examining the weak coupling limit of (1.4) in which the integer $n$ in (1.1) is very large. This corresponds to very small value of $\hbar$ the Planck's constant. Consequently, the functional (1.4) is dominated by the classical part of the action. Hence we evaluate (1.4) as sum of contributions coming from the stationary points which are the solutions of the classical equation of motion (1.7). To proceed one expands the gauge field $A$ around the classical backgrounds $A^{(i)}, A=A^{(i)}+B$, of (1.7), then gauge-fix the action by introducing a pair of anticommuting ghosts $c$ and $c^{*}$. It is straightforward to evaluate (1.4) and find:

$$
W(M, 0)=\exp i n I\left(A^{(i)}\right) \cdot\left(\operatorname{det}\left(K_{1}\right)^{1 / 2} / \operatorname{det}\left(K_{2}\right),\right.
$$

where $K_{1}$ is the kinetic operator of the gauge field $B$, and $K_{2}$ is that of the ghost fields. Further evaluation of the determinants yields the final answer $[6,17]$ :

$$
W(M, 0)=\exp i \eta(0) / 2 \sum_{i} \exp i\left(n+c_{2}(G) / 2\right) \cdot I\left(A^{(i)}\right) \cdot T_{(i)},
$$

which indicates clearly that quantum corrections to (1.1) shifts the integer $n$ to $n+c_{2}(G) / 2$ where $c_{2}(G)$ is the value of the Casimir operator of the group $G$ in the adjoint representation. Therefore, in (2.7) we replace $n$ by $n+c_{2}(G) / 2$, and after rescaling $W$ by $(1 / N) \cdot W$ we obtain (2.10). Note that for $S U(N), C_{2}(G)=2 N$. From (2.7) we deduce the following important property of $W(C, R)$ which is also true for ordinary Yang-Mills gauge theories:

$$
K_{s} W(C, R):=\dot{x}^{\mu}(s) \frac{\delta}{\delta x^{\mu}(s)} W(C, R)=0,
$$

which states that $W$ is reparametrization invariant. 
Equations (2.7)-(2.12) are the basic fundamental quantum equations of the quantized Chern-Simons theory in $d=3$ written in loop space. We would like to find solutions of these equations, however, before we go further on, we would like to comment on (2.10) in connection with the Q.C.D. loop equations in $d=4$ (or $d=3$ ). In addition to the property (2.12) the Eqs. (2.7) and (2.10) are first order variational equations while for Q.C.D. we have second order variational equations in the form $\delta^{2} / \delta x^{2} W(C)^{1}$. Unfortunately $\delta^{2} / \delta x^{2}(s)$ is not well defined and possibly singular $[3,4]$ and this makes the reconstructions of $W(C)$ in Q.C.D. problematic. Luckily enough we do not have this specific problem for Chern-Simons theory, thanks to the $F=0$ field equation. This will enable us to find exact solutions of (2.7) $-(2.10)-(2.12)$ for any value of $N$. Furthermore, in the quantum CS theory the partition function $W$ satisfies a factorization identity for an unknotted and unlinked loops i.e.:

$$
W^{2}\left(M_{3}, C_{1}, R_{1} ; C_{2}, R_{2}\right)=W\left(M_{3}, C_{1}, R_{1}\right) \cdot W\left(M_{3}, C_{2}, R_{2}\right) .
$$

This factorization identity is exact and it is a characteristic feature of topological quantum field theory. This is in contrast to the situation in Q.C.D. where (A) is only valid in the large $N$ limit. One can prove (A) in the following way: We consider the right-hand side (A) and expand perturbatively the expressions (1.5) and (1.9) as presented in (3.1) which is a well defined and acceptable expansion. We will obtain the left-hand side of (A) together with all the contributions corresponding to the Wick's contractions of various orders of the gauge fields coming from $W\left(C_{1}\right)$ and $W\left(C_{2}\right)$. Since the theory we are considering is topological, i.e. there is no local dynamics, therefore it follows that there is not a local propagator for the $A$ gauge field. This implies that all the Wick's contractions are zero and hence we are left with the property (A). This identity will play a fundamental role in linearizing the loop equation (2.10) as we will see shortly.

3a. Renormalization and Regularization of the Quantum Loop Equation. We would like to tackle the divergences that might arise in our solutions to (2.7), and whether we can extract the divergences of $W$. To this extent let us expand $W(C, R)$ to first order in perturbation:

$$
\begin{aligned}
W(C, R) & =\left\{1+\oint_{s_{1}<s_{2}} \Gamma_{\mu v}\left(x_{1}, x_{2}\right) d x_{1}^{\mu} d x_{2}^{v}+\ldots\right\}, \\
\Gamma_{\mu v}\left(x_{1}, x_{2}\right) & =\left\langle\operatorname{tr} A_{\mu}\left(x_{1}\right) A_{v}\left(x_{2}\right)\right\rangle,
\end{aligned}
$$

inserting (3.1) into (2.7) and keeping $W \sim 1$ in the left-hand side of (2.7) to first order approximation we obtain:

$$
\partial_{\mu} \Gamma_{\varrho \sigma}\left(x, x^{\prime}\right)-\partial_{\varrho} \Gamma_{\mu \sigma}\left(x, x^{\prime}\right)=\frac{2 \pi i}{m} \tilde{\varepsilon}_{\mu \varrho \sigma} \delta\left(x-x^{\prime}\right)+\partial_{\sigma} \varphi_{\mu \varrho},
$$

where $\varphi_{\mu \varrho}=-\varphi_{\varrho \mu}$ is an arbitrary tensor. Equation (3.2) can be solved for $\Gamma_{\varrho \sigma}\left(x, x^{\prime}\right)$ and we have:

$$
\Gamma_{\varrho \sigma}\left(x, x^{\prime}\right)=\frac{2 \pi i}{m}\left\{\frac{1}{4 \pi} \tilde{\varepsilon}_{\mu \varrho \sigma} \frac{\left(x^{\mu}(s)-x^{\mu}\left(s^{\prime}\right)\right)}{\left|x(s)-x\left(s^{\prime}\right)\right|^{3}}\right\}+\partial_{[\varrho} V_{\sigma]},
$$

\footnotetext{
${ }^{1}$ This is because the classical field equation of ordinary Yang-Mills theory is $\nabla_{\mu} F^{\mu v}=0$ unlike the Chern-Simons theory which has $F=0$
} 
with $V$ being arbitrary, and $m$ is given by Eq. (2.11). Consistency with (3.2) requires that

$$
\begin{aligned}
\varphi_{\varrho \sigma}\left(x, x^{\prime}\right) & =\partial_{[\varrho} V_{\sigma]}\left(x, x^{\prime}\right):=\left(\partial_{\varrho} V_{\sigma}-\partial_{\sigma} V_{\varrho}\right)\left(x, x^{\prime}\right), \\
\tilde{\varepsilon}_{\mu \varrho \sigma}: & =\frac{1}{N} C_{2}(R) \varepsilon_{\mu \varrho \sigma} .
\end{aligned}
$$

Denote by $W^{(2)}$ the second term in (3.1) we obtain:

$$
\begin{aligned}
& W^{(2)}(C, R)=\frac{2 \pi i}{N \cdot m} C_{2}(R) \chi(C, C)=\cong(C), \\
& \chi\left(C_{1}, C_{2}\right)=\frac{1}{4 \pi} \oint_{C_{1}} \oint_{C_{2}} \varepsilon_{\mu \varrho \sigma} \frac{\left(x^{\mu}-x^{\mu \prime}\right)}{\left|x-x^{\prime}\right|^{3}} d x^{\varrho} d x^{\sigma} .
\end{aligned}
$$

Where $c_{2}(R)$ is the casimir value of the fundamental representation $R$ of the group $G$. If $C_{1} \neq C_{2}$ then $\chi\left(C_{1}, C_{2}\right)$ is the celebrated Gauss linking number which is an invariant in knot theory. Unfortunately, the integral (3.5b) contains the situation where $C_{1}=C_{2}=C$ and then $\chi$ is ill-defined and needs a proper regularization. The most appropriate one which respects general covariance is the manifold-splitting regularization scheme $[8,6]$. Briefly this amounts to thickening the knot $C$ to a ribbon; thur turning a space-time curve into a space-time ribbon whose edges are the initial curve $C$ and a separate curve $C_{\varepsilon}$, away an infinitesimal distance $\varepsilon \ll 1$ from $C$. Now we can apply safely Gauss's formula for $\chi\left(C, C_{\varepsilon}\right)$. Then we extract for $\varepsilon \ll 1$ an independent topological invariant; namely the linking of the two edges of the ribbon. This invariant will be the self-linking number of $C$ itself. The curve $C_{\varepsilon}$ is parametrized by $(x(s), \mathbf{n})$ where $\mathbf{n}(s)$ is the unit normal vector to the curve $C, x(s)$. We take $x(s)=x(s)+\varepsilon n(s)$. Inserting this into $\chi\left(C, C_{\varepsilon}\right)$, and extracting the $\varepsilon$ independent term and then take the limit $\varepsilon \rightarrow 0$, one obtains:

$$
\chi_{\mathrm{reg}}(C, C)=\frac{1}{2 \pi} \cdot \int_{0}^{L} V(s) d s ; \quad V(s)=\mathbf{n} x d \mathbf{n} / d s,
$$

where $V(s)$ is the Dirac potential on a sphere $\mathbf{e}^{2}=1$ with $\mathbf{e}_{1}=\partial \mathbf{x} / \partial s ; \mathbf{e}_{2}=\mathbf{n}$; $\mathbf{e}_{3}=\mathbf{e}_{1} x \mathbf{e}_{2}$ being the Serret-Frenet moving frame, and $L$ is the length of the loop $C$. Therefore,

$$
W^{(2)}(C, R)=\frac{2 \pi i}{N \cdot m} C_{2}(R) \cdot \frac{1}{\varepsilon^{2}} \chi(C, C),
$$

where "reg" stands for regularized. A far more interesting piece of calculation, which we are not going to present in this paper, is to do the fourth order in perturbation $\left(g^{4}\right.$ in the Yang-Mills coupling constant).

From a very tedious exercise we have found that the divergent part of $W$ at the fourth order takes the form:

$$
W^{(4)}(C, R) \equiv\left\{\frac{2 \pi i}{N \cdot m} C_{2}(R) \frac{1}{\varepsilon^{2}} \chi(C, C)\right\}^{2}+\underset{\text { as } \varepsilon \rightarrow 0}{\text { finite }} \text { terms } .
$$

This remarkable result strongly suggests that all the divergent terms of $W(C)$ can all be summed up in the form:

$$
W\left(M_{3}, C, R\right)=\hat{\Phi}(C, R) W_{\text {ren }}\left(M_{3}, C, R\right),
$$




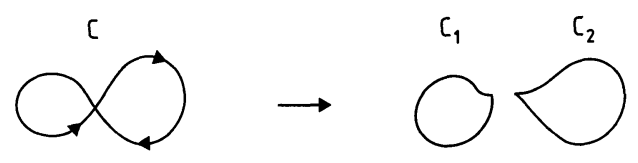

Fig. 1

$x(S)=y$

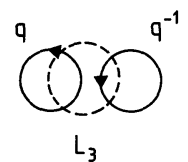

$W\left(S^{3}, C^{2}\right)$

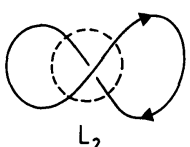

$W\left(S^{3}, C\right)$

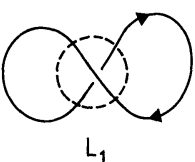

$W\left(S^{3}, C\right)$

Fig. 3

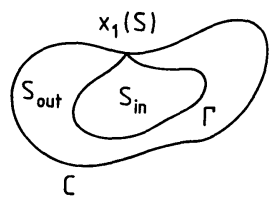

where

$$
\hat{\Phi}(C, R)=\exp \frac{2 \pi i}{N \cdot m} C_{2}(R) \cdot \frac{1}{\varepsilon^{2}} \chi(C, C)
$$

We recognize $\hat{\Phi}$ as the torsion of the curve $C$; and it was introduced recently by Polyakov [9] within the context of abelian Chern-Simons theory applications to high temperature superconductivity. $W_{\mathrm{REN}}(C)$ stands for the renormalized, finite value of the partition function in which the coupling constant is being renormalized. Let us remark that Eq. (3.9) is an extension of Polyakov's result to the non-abelian theory in the sense that $m=n+C_{2}(G) / 2$ contains the value of the Casimic operator of the group $G$. The answer (3.7), (3.9) depends on the choice of framing of the knot $C$. For example, if we allow $C$ to twist say $\theta$ units around $C$ then the solutions (3.7) $-(3.9)$ are shifted by a phase factor exp $\cdot 2 \pi i \cdot \theta \cdot h[6]$, where $h$ is the conformal weight of a primary field living in $1+1$ dimensions (which is the surface $S$ that comes out in the solution of $W[C, R]$ ). Therefore, it is left to determine $W_{\text {REN }}[C, R]$. The result (3.8) was of utmost importance in proving that the loop equation (2.10), in its linear version (3.11) yields precisely the skein relation of knot theory [18].

The expression (2.10) as it stands suffers from ultraviolet divergences due to the presence of the $\delta$-function. Therefore, (2.10) must be regularized by smearing the $\delta$-function. The regularized loop equation would hold for any arbitrary loop, and there would be no distinction between intersecting and non-intersecting loops. An appropriate method to work out the regularized version of the loop equations (2.7) and (2.10) is to introduce the proper time $T=1 / \Lambda^{2}$ coordinate. The regularized loop equation takes the following form (Fig. 5):

$$
\begin{aligned}
\frac{\delta}{\delta \sigma^{\mu}(s)} W(C)= & \frac{2 \pi i}{m} \oint_{C_{x x}} d y^{\mu} \int_{\gamma(0)=x(s)}^{\gamma(T)=y} D \gamma \exp -\frac{1}{2} \int_{0}^{T} d t \dot{\gamma}^{2} \\
& \times\left\{W^{2}\left(C_{x y} \gamma_{y x}, C_{y x} \gamma_{x y}\right)-\frac{1}{N} W(\gamma) W\left(C_{y y}\right)\right\} .
\end{aligned}
$$


Equation (3.10a) is obtained from the regularized measure

$$
\begin{aligned}
\frac{\delta}{\delta \sigma^{\mu}(s)} W(C) & =\frac{2 \pi i}{m} \oint_{C_{x x}} d y^{\mu} U_{x y}, \\
U_{x y}^{a b} & =\int_{\gamma(0)=x(s)}^{\gamma(T)=y} D \gamma\left(\exp -\frac{1}{2} \int_{0}^{T} d t \dot{\gamma}^{\mu} \dot{\gamma}_{\mu}\right) \operatorname{tr}\left\{T^{a} \psi\left(C_{x y} \gamma_{y x}\right) T^{b} \psi\left(C_{y x} y_{x y}\right)\right\},
\end{aligned}
$$

and the use of Eqs. (2.1) and (2.5). In the limit where the proper time goes to zero we reproduce Eqs. (2.7) and (2.10) as a consequence of the following relation:

$$
f(x, y) \delta^{a b}:=\int_{\gamma(0)=x(s)}^{\gamma(T)=y} D \gamma \exp -\frac{1}{2} \int_{0}^{T} d t \dot{\gamma}^{2} \operatorname{tr}\left(T^{a} T^{b}\right)=\delta^{a b} e^{T \cdot \square} \delta^{3}(x-y) .
$$

It is this equation that we will examine for well defined solutions, supplemented by the fact that $W$ is reparametrization invariant (2.12). Notice that if the contour is smooth the left-hand side of (2.7) is zero, and we get Eq. (2.2) whose solution is an arbitrary function that is path independent. If the contour has self intersections then the left-hand side of (2.7) survives. The partition function $W$ satisfies a further interesting property which can be deduced from a close inspection of Figs. 4 and 6 , taking into consideration the choice of framing of the loop $C$ which we have discussed earlier:

$$
\oint_{C_{x x}} d y f(x, y)\left\{W\left(C_{x y} \gamma_{y x}\right) W\left(C_{y x} \gamma_{x y}\right)-W(\gamma) W\left(C_{y y}\right)\right\}=0 .
$$

Fig. 4

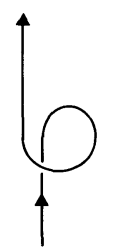

L.

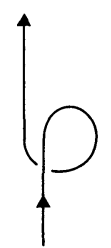

L.

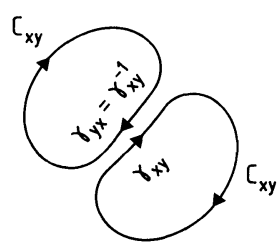

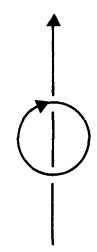

$L_{0}$

Fig. 5

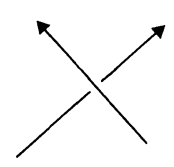

$K_{+}$

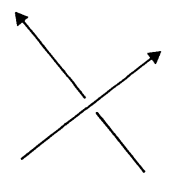

K.

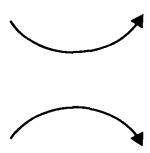

$K_{0}$

Fig. 6 
Using this property, together with the identity (A) the regularized loop equation (3.10a) takes the following final linear form for the oriented link diagrams in Figs. 4 and 6 which are identical except near a point where they have positive, negative, and zero crossings [19]:

$$
\delta W(C)=\frac{2 \pi i}{m}\left(1-\frac{1}{N}\right) \oint_{C_{x x}} d y^{\mu} \delta \sigma_{\mu}(x(s)) f(x, y) W(\gamma) W(C) .
$$

It is this version of the loop equation, together with the property (3.8) that yields precisely the linear skein relation of knot theory [18] as we will briefly outline in the next subsection.

The above equation indicates clearly that CS theory is an exactly soluble theory. The linearity of (3.11), and the fact that it is a first order loop equation is a unique feature of the topological gauge quantum field theory we are considering. We recall that a similar situation occurs for the 3-d Ising model [10]. As a justification of our claim, we will present a simple solution of (3.11) and (2.12). In the next subsection we will give a more physically interesting solution which might be of potential importance in the theory of knots. Consider the following vector field:

$$
\sigma_{\mu}:=\frac{1}{2} \varepsilon_{\mu \varrho \sigma} \sigma^{\varrho \sigma} ; \quad \sigma^{\varrho \sigma}=\oint_{C} x^{\varrho}(s) d x^{\sigma}(s) .
$$

If the loop $C$ is smooth, it is easy to show that

$$
K_{s} \sigma_{\mu}=0,
$$

where the $K_{s}$ operator is defined in (2.12), i.e. $\sigma$ is reparametrization invariant; however, if the loop intersects itself say at the point $x\left(s_{1}\right)=x\left(s_{2}\right)=x(s)$, Fig. 1 . Then (3.12) breaks down at the point of intersection which has been fixed a priori. Therefore, to restore (3.12) we must subtract the contribution of $\sigma$ at the point of interaction. The correct subtraction is given by:

$$
\eta_{\mu}=\frac{1}{2} \varepsilon_{\mu \varrho \sigma} \oint_{C_{x x}} \delta^{3}\left(x(s)-x\left(s^{\prime}\right)\right) x^{e}(s) \dot{x}^{\sigma}\left(s^{\prime}\right) d s d s^{\prime},
$$

which is similar to $\sigma^{\mu}$ with the exception of the $\delta$-function. The presence of the $\delta$-function is crucial in the following sense: if the loop $C$ does not intersect itself at the point $x(s)$ then [3]:

$$
\delta^{(d)}\left(x(s)-x\left(s^{\prime}\right)\right)=0 \Leftrightarrow \eta_{\mu}=0 .
$$

However, if $C$ itself intersects $s$ at $x\left(s_{1}\right)=x\left(s_{2}\right)$ then $\eta^{\mu}$ receives a non-zero contribution from the neighbourhood of the self intersection point. To calculate this contribution we need to determine the $\delta$-function near the point of the interaction. We can do that by taking the following ansatz of $\delta$ :

$$
\delta^{(d)}\left(x(s)-x\left(s^{\prime}\right)\right)=\lim _{\varepsilon \rightarrow 0} \varepsilon \cdot \frac{1}{\left|x(s)-x\left(s^{\prime}\right)\right|^{d-\varepsilon}}
$$

and calculate it between two test functions. For $d=3$ we obtain [7]:

$$
\delta^{3}\left(x(s)-x\left(s^{\prime}\right)\right)=a_{1}\left\{\dot{\delta}\left(s-s_{1}\right) \delta\left(s^{\prime}-s_{2}\right)-\delta\left(s-s_{1}\right) \dot{\delta}\left(s^{\prime}-s_{2}\right)+s \leftrightarrow s^{\prime}\right\},
$$


where $a_{1}$ is just a finite constant. Substituting (3.15) into (3.12) we obtain the contribution of $\sigma^{\mu}$ at the point of interaction. Denote by:

$$
Q^{\mu}=\sigma^{\mu}-\eta^{\mu}
$$

then for $C$, smooth, self intersecting or knotting we have (Fig. 1):

$$
K_{s} Q^{\mu}=0
$$

and hence

$$
W(C)=\lambda \int_{M_{3}} \mathscr{D} g \exp -\left(Q^{\mu} Q_{\mu}\right)^{1 / 2} / d^{\prime} .
$$

is a solution of Eq. (2.12), where $\lambda$ is an arbitrary constant and $\mathscr{D} g$ is the measure of the 3-metric on $M_{3}$. With appropriate fixing of $\lambda$ it is straightforward to show that (3.18) satisfies (3.11). Next, we will present another appealing solution to Eq. (3.10a) which would be of considerable importance to two-dimensional (conformal) field theory models.

3b. Fermi String Theory, and the Linear Knot Skein Relation. At first sight it would be strange to think of any connection between CS theory in $d=3$ and Fermionic strings, however, it seems that such a connection is indeed there at least between Q.C.D. and strings [3-5]. The basic line of thought is to imagine that the Wilson loop $C$ is a line being created by a fermionic field at a reference point $x_{0}$, and then closing this line by introducing an anti-fermionic field at $x_{0}$. The trajectory of these (anti)fermionic fields would be a surface $S$ embedded in $M$, whose boundary is the loop $C$. The crucial question is whether or not the surface $S$ exists for an arbitrary three-dimensional manifold $M$. The answer is certainly negative in general, however, as we shall discuss in Sect. 4 there is a whole class of non-simply connected 3-manifolds in which any loop $C$ embedded in it can be thought of as the boundary of a surface $S$ in $M_{3}$. In this section we will consider the case of $S^{3}$ in which $S$ definitely exists and leave the generalization to Sect. 4 . Therefore, our goal is to show that there exists a quantum string theory defined on $S$ that is equivalent to the quantum CS theory.

Let $S$ be the surface on $M_{3}$ whose boundary is the loop $C$ (we allow $S$ to have self-intersections), and has a given induced metric

$$
g_{\alpha \beta}(\sigma, \tau)=\varphi(\sigma, \tau) \eta_{\alpha \beta}:=\partial_{\alpha} x^{\mu}(s) \partial_{\beta} x_{\mu}(S)
$$

in the conformal gauge, where $\sigma$ and $\tau$ are the internal coordinates on the surface $S$. The conformal factor $\Phi$ is reparametrization invariant; this follows from

$$
\frac{\delta}{\delta x^{\mu}(s)} \varphi(\sigma, \tau)=\delta\left(\sigma-\sigma_{0}\right) \delta\left(\tau-\tau_{0}\right) \varepsilon_{\mu \varrho \sigma} \dot{x}^{\varrho}(s) v^{\sigma}(s),
$$

where $t^{\mu \nu}=\varepsilon^{\mu v \varrho} v_{\varrho}$ is given in terms of the surface area and it is a tangential antisymmetric tensor to $S$ :

$$
d \sigma^{\mu \nu}=t^{\mu \nu} \sqrt{\mathrm{g}} d^{2} \sigma .
$$

Equation (3.19) can be expressed in terms of $\delta \sigma^{\mu}$ [cf. Eq. (2.9)] as

$$
\frac{\delta \varphi}{\delta \sigma^{\mu}}=v^{\mu} \delta\left(\sigma-\sigma_{0}\right) \delta\left(\tau-\tau_{0}\right)
$$


let $\psi_{\lambda}^{\alpha i}(\sigma, \tau) ; \alpha=1,2 ; \lambda= \pm 1$ be a bispinor on $S$ (an elfin) which satisfies the following boundary conditions $(i=1, \ldots, N)$ :

$$
\begin{array}{cl}
\sigma_{3} \psi_{\lambda}=\lambda \psi_{\lambda} ; & \bar{\psi}_{\lambda} \sigma_{3}=\lambda \bar{\psi}_{\lambda}, \\
\psi_{\lambda}^{R, L}=\frac{\left(1 \pm \lambda \sigma_{3}\right)}{2} \psi_{\lambda} ; & \bar{\psi}_{\lambda}^{R, L}=\bar{\psi}_{\lambda} \frac{\left(1 \pm \lambda \sigma_{3}\right)}{2}
\end{array}
$$

at the boundary of $S . \lambda \sigma_{3}$ describes the orientations of the loop $C$. The spinor $\psi$ is $O(3)$ invariant as a consequence of (3.22). Equation (3.22b) describes the oriented states in which the left-handed states will be occupied at the boundary. The dynamics of $\psi$ can be obtained from the action principle

$$
I_{\psi}=\int d^{2} \sigma\left(e^{2 \varrho} \bar{\psi}_{\lambda}^{i} \sigma^{\alpha} e^{-\varrho} \nabla_{\alpha} \psi_{\lambda i}+\sqrt{\varphi} e^{\varrho} \tilde{M} \bar{\psi}_{\lambda}^{i} \psi_{\lambda i}-\frac{1}{6 \pi} \partial^{\alpha} \varrho \partial_{\alpha} \varrho\right),
$$

where $\varrho$ is an external field, $\sigma^{\alpha}$ are of the pauli matrices, and $\omega_{\alpha}\left(\nabla_{\alpha}=\partial_{\alpha}+i \sigma_{3} \omega_{\alpha}\right)$ is the spin connection. It has been shown that the quantum field theory of $I_{\Psi}$ is $\varrho$ independent, and the presence of $\partial_{\alpha} \varrho \partial_{\alpha} \varrho$ in $I_{\psi}$ is crucial for this to be true [5]. The partition function of $I_{\psi}$ is given by:

$$
Z[S]=\int D \psi D \bar{\psi} \exp I_{\psi}=\exp \left(-\frac{1}{6 \pi} \int d^{2} \sigma \partial_{\alpha} \varrho \partial_{\alpha} \varrho\right) \Delta_{+} \Delta_{-}
$$

and

$$
\begin{aligned}
& \Delta_{\lambda}=\operatorname{Det}_{\lambda}\left(i D_{e}+\tilde{M} \sqrt{\varphi} e^{-\varrho}\right), \\
& D_{e}=\sigma^{\alpha} e^{-\varrho}\left(-i \partial_{\alpha}+\sigma_{3} \omega_{\alpha}\right),
\end{aligned}
$$

with the trace being taken over the boundary conditions $\sigma_{3}= \pm 1$, and the irreducible representation of the gauge group $G$. It is straightforward to show using the fact that $Z$ is $\varrho$ independent and Eq. (3.19) that $Z$ is reparametrization invariant:

$$
K_{S} Z[S]:=\dot{x}^{\mu}(s) \frac{\delta}{\delta x^{\mu}(s)} Z[S]=0 .
$$

Consider Fig. 3 in which we have a loop $\Gamma$ that has an opposite orientation to $C$. This can be achieved by choosing the opposite boundary conditions to (3.22a), namely, we require $\Psi^{R}=\bar{\psi}^{R}=0$, at $\Gamma^{-1}$. Consequently, the string propagator $Z$ can be shown to satisfy a factorization relation. Let $\gamma_{x y}\left(\gamma_{x y}^{-1}=\gamma_{y x}\right)$ be an infinitesimal wire that connects a point $x$ to a point $y=x+\delta x$. An explicit evaluation of (3.24) reveals that $Z$ satisfies the following relations $[5,7]$ :

$$
Z[C]=\int D \Gamma_{\|} Z\left[C_{\|} \Gamma_{\|}\right] Z\left[\Gamma_{\|}^{-1}\right] \exp -i \alpha l_{\Gamma},
$$

where $\alpha$ is a constant parameter, and $\Gamma_{\|}$is a closed path of length $l$ on the surface $S$. Under the action of the area derivative (3.21) only the fermionic bilinear term in the quantum theory of (3.23) contributes. This amounts to creating a fermionantifermion pair at the boundary of $S$ that forms the ends of a closed path $\Gamma_{\|}$. This path touches the boundary at a point $x_{1}(s)$ and hence splits the surface $S$ into two 
pieces $S_{\text {in }}$ and $S_{\text {out }}$. Consequently, it follows from (3.27) and (3.21) that (Fig. 5):

$$
\frac{\delta}{\delta \sigma^{\mu}(s)} Z[C]=-\frac{i \alpha}{2} \int D \gamma_{x y} v^{\mu}(x(s)) \exp -\alpha l_{\gamma_{x y}} Z\left[C_{x y} \gamma_{y x}\right] Z\left[C_{y x} \gamma_{x y}\right]
$$

with $l_{x y}$ being the length of the wire $\gamma_{x y}\left(l_{x y}=\int_{\gamma_{x y}} d^{2} \sigma \varphi^{1 / 2}\right)$. The presence of the wires $\gamma_{x y}$, and $\gamma_{y x}$ is to make the loops $C_{x y}$ and $C_{y x}$ closed and continuous. Comparing Eqs. (3.28) and (3.10a) together with the identity (A) leads to the immediate conclusion that $Z[C]$ is equivalent to $W[C]$. In fact, $W=Z \cdot \operatorname{expi\alpha l_{C}}$, the exponential term has the effect of producing the linear term in the loop equation (3.10a) due to the following variational equation:

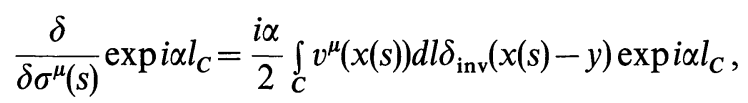

where the invariant $\delta$-function is

$$
\delta_{\text {inv }}(x(s)-y)=\varphi^{-1} \delta\left(\sigma-\sigma_{0}\right) \delta\left(\tau-\tau_{0}\right)
$$

with $x(s)$ being a point at the boundary.

It is of utmost importance to realize that $Z[C]$ is the regularized solution for $W(C)$. Note that if we shrink the wires $\gamma_{x y}$ to zero in (3.28) we obtain a $\delta$-function. Therefore, we propose the following regularized solution for the loop equation (3.10a):

$$
W\left(M_{3}, C, R\right) / W\left(M_{3}\right)=\hat{\Phi}(C, R) \cdot \sum_{\substack{S \in M_{3} \\ \partial S=C}} Z[S] / Z[S, \tilde{M}=0] \cdot e^{i \alpha l_{C}},
$$

where $\varphi$ is the path torsion (3.10). The constant $\alpha$ can be fixed by inserting (3.30a) into (3.10a) and comparing coefficients:

$$
\alpha=-\frac{4 \pi i}{m} ; \quad \tilde{M} / l_{C}=\frac{4 \pi}{m} .
$$

Equation (3.30c) provides the connection between the 2-d Fermionic string theory and the CS theory (1.9). Remarkably we find that the string mass $M$ per unit length is quantized in order of $h$. It is straightforward to prove the identity (A) explicitly using the ansatz (3.30a). It is important to mention that knots are included in our theory provided that the surface $S$ is self-intersecting with possible singular points (these are the endpoints of the lines of self-intersections). In this case our problem reduces to the study of the behaviour and the properties of the Dirac operator induced on $S$ at the singular points $\left\{t_{k}\right\}$. One particular approach which we favour is to isolate the singular points by discs $\left\{D_{k}\right\},|z|<\varepsilon_{k}$ centered at $t_{k}$ and evaluate (3.25) on $S-\left\{D_{k}\right\}$. The theory (3.24) with singular self-intersecting surfaces can be obtained as a limit of the theory on $S-\left\{D_{k}\right\}$ when $\varepsilon_{k} \rightarrow 0$. We will present the details elsewhere. However, for the prove to come next we will assume the limit exists and proceed formally.

Let $M_{1}$ and $M_{2}$ be two 3-dimensional manifolds and $W\left(M_{1}, L_{1}\right) ; W\left(M_{2}, L_{2}\right)$ be their corresponding partition functions with $L$ being the link of the loop $(s)(C, R)$. Suppose we want to form a new 3-dimensional manifold $M=M_{1}+M_{2}$. A standard procedure would be to cut a ball from $M_{1}$ and $M_{2}$. Then we glue $M_{1}$ and 
$M_{2}$ along the boundaries of the two balls being removed (which are $S^{2}$ ) after identifying them. We obtain a new manifold $M$ with a closed hole inside, which we can eliminate by filling it with $S^{3}$. From Eq. (3.30) it follows that:

$$
\begin{aligned}
& W\left(M_{1}, L_{1}\right) \rightarrow W\left(M_{1}, L_{1}\right) \cdot W\left(S^{2}, p_{1}, \ldots, p_{n}\right), \\
& W\left(M_{2}, L_{2}\right) \rightarrow W\left(M_{2}, L_{2}\right) \cdot W\left(S^{2}, q_{1}, \ldots, q_{n}\right),
\end{aligned}
$$

where $P_{1}, \ldots, P_{m}$ (respectively $q_{1}, \ldots, q_{M}$ ) are the cuts of $L_{1}$ (respectively $L_{2}$ ) at the boundary of $S^{2}$. Under $G$ transformation we identify the points $\left\{p_{1}, \ldots, p_{m}\right\}$ with $\left\{q_{1}, \ldots, q_{m}\right\}$ with no intersection, i.e. they are elements of the irreducible representation of $G$. In this way we obtain a new link $L=L_{1}+L_{2}$ on $M=M_{1}+M_{2}$ which has no component on $S^{3}$. Using (3.31) Witten [6] showed that $W$ satisfies the following factorization identity $\left(\gamma\right.$ is an unknotted loop on $\left.S^{3}\right)$ :

$$
W\left(M_{1} \cup M_{2}, L_{1}+L_{2}\right) \cdot W\left(S^{3}, \gamma\right)=W\left(M_{1}, L_{1}\right) \cdot W\left(M_{2}, L_{2}\right) .
$$

We can prove (3.32) using the solution (3.30b) and the fact that $Z$ does factorize on two glued surfaces $S_{1}$ and $S_{2}$ (we have chosen the cut of $M$ to pass through the surface $S$ ):

$$
Z\left(S_{1} \cup S_{2}\right) \cdot Z(D)=\sum_{i} Z\left(S_{1}, B_{i}\right) \cdot Z\left(S_{2}, B_{i}\right),
$$

where $D$ denotes the disc and $B_{i}$ are fixed boundary conditions on the loop $C^{\prime}$, the boundary of $S_{1}$ and $S_{2}$, respectively. Notice that $S$ which is $S_{1} \cup S_{2}$ is cut along $C^{\prime}$. The generalization of the identity (3.32) to non-simply connected 3-d manifolds cut along an arbitrary compact Riemann surface will be presented in Sect. 4.

Finally, we would like to calculate $W^{(1)}\left(S^{3}, L\right)$. We will do that on the basis of examining the linear loop equation (3.11) within the context of knot theory. We will do a brief presentation of this very interesting connection. The full technical details can be found in [18]. We go back to (3.11) we recognize that:

$$
\lim _{\gamma \rightarrow 0} \oint_{C_{x x}} \delta \sigma^{\mu}(x(s)) d y_{\mu} f(x, y) W(\gamma)=1
$$

is a dimensionless quantity, and therefore, we normalize $W(\gamma)$ such that the lefthand side of (3.33) is one. Then the variational loop equation takes the simple form:

$$
\delta W(C)=2 \pi i / m(1-1 / N) W(C) .
$$

Consider the diagrams in Fig. 4 in which we have three knots, $L_{+}, L_{-}, L_{0}$ [19], with overcrossing, undercrossing, and zero crossing, respectively. The loop equation (3.34) encodes the variation or deformation of the knot $L_{+}$to the knot $L_{-}$. Therefore, we deduce from (3.34) that:

$$
W^{-1} \delta W=\delta \ln W=\ln W\left(L_{+}\right)-\ln W\left(L_{-}\right)=2 \pi i / m(1-1 / N),
$$

i.e.

$$
W\left(L_{+}\right) / W\left(L_{-}\right)=\exp 2 \pi i / m(1-1 / N) .
$$

Using Eqs. (3.8) and (3.9) we can obtain the ratio of the renormalized partition function $W_{\mathrm{REN}}(C):=\hat{W}(C)$ :

$$
\hat{W}\left(L_{+}\right) / \hat{W}\left(L_{-}\right)=\exp 2 \pi i / m\left(1-1 / N+2 C_{2}(R) / N\right) .
$$


I

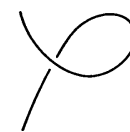

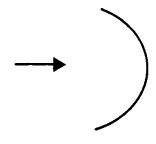

II

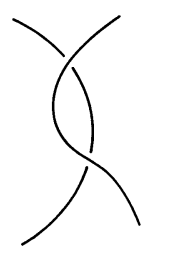

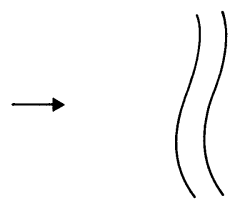

and

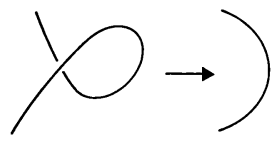

III
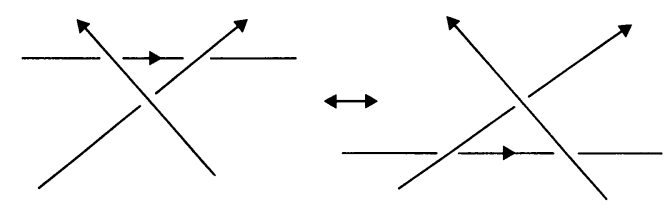

Fig. 7

A similar result would hold for the diagrams $K_{+}$and $K_{-}$in Fig. 6 . On the other hand, from the factorization identity (A) and inspection of Fig. 4, it follows that:

$$
\hat{W}\left(L_{+}\right) \cdot \hat{W}\left(L_{-}\right)=\left\{\hat{W}\left(L_{0}\right) \cdot \hat{W}\left(C_{0}\right)\right\}^{2},
$$

where $\hat{W}\left(C_{0}\right)$ is the partition function of an unknotted and unlinked loop $C$. The second equality in (3.36) follows from the particular choice of framing of the loop $C$, we adopted before. From (3.35b) and (3.36) we can solve uniquely for $\hat{W}\left(L_{+}\right)$and $\hat{W}\left(L_{-}\right)$. We obtain:

$$
\begin{gathered}
\hat{W}\left(L_{+}\right)=\exp \pi i / m\left(1-1 / N+2 C_{2}(R) / N\right) \cdot \hat{W}\left(L_{0}\right) \cdot \hat{W}\left(C_{0}\right), \\
\hat{W}\left(L_{-}\right)=\exp -\pi i / m\left(1-1 / N+2 C_{2}(R) / N\right) \cdot \hat{W}\left(L_{0}\right) \cdot \hat{W}\left(C_{0}\right) .
\end{gathered}
$$

As an application we consider the group $G=S U(N)$ in the fundamental representation $R$, in which case $c_{2}(R)=1 / 2\left(N^{2}-1\right)$; then it is straightforward to deduce from (3.37) and Fig. 6 that:

$$
\begin{gathered}
q^{N / 2} \hat{W}\left(K_{+}\right)-q^{-N / 2} \hat{W}\left(K_{-}\right)=\left(q^{1 / 2}-q^{-1 / 2}\right) \hat{W}\left(K_{0}\right) \cdot \hat{W}\left(C_{0}\right), \\
q=\exp 2 \pi i / m,
\end{gathered}
$$

which together with (3.37) constitute the full exact generalized linear skein relation of knot theory in which the renormalized partition function $W_{\text {REN }}$ of the CS theory plays the role of the Laurent polynomial $P(a, b)$ function. The parameters $a$ and $b$ are integer numbers. In our case $a=q^{N / 2}$, and $b=q^{1 / 2}-q^{-1 / 2}$. We would like to mention that not all knot invariants satisfy a linear relation of the form (3.38), for example diagrams of Reidemeister type II and III [19] (Fig. 7). Such knot invariants must satisfy the non-linear regularized loop equation (3.10a). As an application of the skein relation (3.38) we consider again Fig. 2 in which the loops $L_{1}, L_{2}, L_{3}$ are unknotted and unlinked. Their corresponding renormalized partition functions satisfy the skein relation (3.38). However, an inspection of the diagrams of $L_{0}, L_{3}$ in Figs. 2 and 6 reveals that:

$$
W\left(L_{3}\right)=\left\{W\left(K_{0}\right) \cdot W\left(C_{0}\right)\right\}^{2}
$$


for unknotted and unlinked loops. Therefore, Eq. (3.36) reads:

$$
W\left(L_{3}\right)=W\left(L_{1}\right) \cdot W\left(L_{2}\right),
$$

but $W\left(L_{1}\right)$ is equal to $W\left(L_{2}\right)$, and hence we deduce from (3.39) and the skein relation (3.38) that:

$$
W\left(L_{1}\right)=W\left(L_{2}\right)=\left(q^{N / 2}-q^{-N / 2}\right) /\left(q^{1 / 2}-q^{-1 / 2}\right) .
$$

This formula was first derived in [6] for the expectation value of the Wilson loop. It is nothing but the Jones polynomial on $S^{3}$ which is an invariant of knot theory.

Now we would like to derive (3.40) in terms of the characters of $S U(2)$. This would seem plausible since our manifold is $S^{3}$. From the factorization property (A) it is straightforward to find that:

$$
W\left(S^{3}, C\right)=\left\{W^{2}\left(S^{3}, C^{2}\right)\right\}^{1 / 2} .
$$

On the other hand, consider Fig. 2. If we associate $q$ with the first circle then we must associate $q^{-1}$ with the other one or visa-versa. An important property that should be satisfied by $W^{2}\left(S^{3}, C^{2}\right)$ is its invariance under change of orientation of the loops $C^{2}$. In other words $W^{2}$ should be invariant under the discrete transformation $q \rightarrow q^{-1}$. The means that $W^{2}$ must be of the general form:

$$
W^{2}\left(S^{3}, C^{2}\right)=\chi(q) \chi\left(q^{-1}\right) .
$$

In particular, for $S^{3}, \chi(q)$ is nothing but the character of $S U(2)$ in the $N$ dimensional representation of $S U(N)$ as we will demonstrate shortly. Evaluating (3.42) gives

$$
\chi(q)=\operatorname{tr} q^{x}=\sum_{r=0}^{N-1} q^{r}=\left(1-q^{N}\right) /(1-q),
$$

i.e.

$$
W^{2}\left(S^{3}, C^{2}\right)=\left(1-q^{N}\right)\left(1-q^{-N}\right) /(1-q)\left(1-q^{-1}\right),
$$

from which we deduce that $W\left(S^{3}, C\right)$ is equal to $\pm W\left(L_{1}\right)$ in (3.40). In [6] Eq. (3.40) was derived as a consequence of manifold surgery and the knowledge of the space of conformal blocks of four point functions in $S^{2}$. It is very interesting to note that our method reveals the fact that the string partition function and hence (3.40) is nothing but the character of $S U(2)$ for an unknotted loop on $S^{3}$ :

$$
W\left(S^{3}, C\right)=\chi_{l}(\theta),
$$

with the following identifications:

$$
N=2 l+1, \quad \theta=2 \pi / m .
$$

It is clear that the rotations are fractional $S U(N)$-valued angles which correspond precisely to Crystal point groups. These groups are finite subgroups of $S U(2)$ that leave a $n$-gon invariant [13]. Now I would like to demonstrate why Eqs. (3.43) and (3.44) are true. Since we are calculating the expectation value for an unknotted loop on a simply connected manifold this means that we can deform the loop to a point and therefore, we can effectively reduce our calculation to the expectation value of the identity, which must depend only on the manifold $S^{3}$ and 
the symmetry group $S U(N)$. On the other hand, we know that the character of $S U(2)$ can be expressed as a Feynman path integral over $S^{3}$. Therefore, the Feynman path integral of the $S U(N)$ gauge Chern-Simons theory on $S^{3}$ can be partitioned by choosing the dimension of the center of $S U(N)$ to be the same as the dimension of the irreducible representation of $S U(2)$. Hence we expand the partition function of the identity in the center of $S U(N)$ with elements that fall into the Irred. Reps. of $S U(2)$, but $S U(N)$-valued:

$$
\chi(q)=\langle\mathbb{I}\rangle=\int \mathscr{D} A \exp i I_{\mathrm{cs}}=q^{N-1 / 2}+q^{N-3 / 2}+\ldots+q^{-(N-1) / 2},
$$

where the angles $\theta=2 \pi / m$ appear as in (3.37) as a consequence of the identity (2.6), and the expansion in (3.46) is implied in the solution (3.30a). We observe the lefthand side of (3.46) as nothing but the character of $S U(2)$ with rotations and the dimension of the irred. reps. being given by (3.45).

\section{Generalization}

We would like to generalize our previous constructions to non-simply connected 3-d manifolds, though this is straightforward, it is nevertheless subtle and nontrivial. The vacuum states that diagonalize the transition amplitudes $W(C, R)$ are classified by the homotopy classes $[M, G]$ of maps from $M$ into the internal symmetry group $G$. For non-simply connected manifolds $M_{3}$ these homotopy classes are given by the first and the third cohomology groups of $M$ :

$$
[M, G]=H^{1}\left(M, \pi_{1}(G)\right)+H^{3}\left(M, \pi_{3}(G)\right)
$$

and therefore, we have two topological quantities of interest. One is the secondary winding number $n$ which is specified by the cohomology group $H^{3}\left(M, \pi_{3}(G)\right) \simeq Z$ (for simple non-abelian Lie groups $G$ and orientable $M$ ). This is the integer $n$ we encountered in the previous sections. The other topological quantity is called the primary winding number $h$ and it is an element of $H^{1}\left(M, \pi_{1}(G)\right)$ [11]. In general, $g$ and $n$ are independent except for the special case of $G=S O(3)$ where they are related; one being the cube of the other $\left(n \bmod 2=g^{3}\right)[11]$. We will discuss the implications of $H^{1}$ on the solution (3.31) later on.

The solutions of the field equation (1.7) are classified by principle flat $G$-bundles over $M$ and they are constructed by forming equivalence relations of the form $(z, U) \simeq\left(e z, h^{-1}(e) U\right)$ on $M x G$ where $e$ belongs to $\pi_{1}(M), h$ is a homomorphism from $\pi_{1}(M)$ into $G$, and $M$ is the universal covering space of $M$. The bundle $M x_{h} G$ is non-trivial as an associated $\pi_{1}(M)$-bundle (though it might be trivial as a principle $G$-bundle), which means that there exist globally well defined flat connections $A_{h}$ that are classified by the $\operatorname{HOM}\left(\pi_{1}(M) \rightarrow G\right)$ and they are patched together non-trivially. Locally these connections take the form:

$$
A_{h}(x)=U(x) d U(x)^{-1} .
$$

If $\pi_{1}(M)=0$ i.e. $M$ is simply connected then $M x G$ is a trivial bundle and an acceptable globally well defined solution of (1.7) is

$$
A=0 \text {. }
$$


In this case the Wilson loop vanishes classically. Quantum mechanically it does not, however, we perform our quantization in the classical background (4.3). On the other hand, if $\pi_{1}(M) \neq 0$ then we must quantize (1.8) around the non-vanishing classical backgrounds $A_{h}$. The quantization of (1.8) yields the following effects: The action being a 3-form will only react to $H^{3}\left(M, \pi_{3}(G)\right)$ under the finite gauge transformation (1.3) and hence as before the invariance of the EXPiI $I_{c \cdot s}$ will give the previous quantization condition $1 / \hbar=n \in Z \simeq H^{3}\left(M, \pi_{3}(G)\right)$. On the other hand, the Wilson loop being the exponent of a 1 -form will only react to $H^{1}\left(M, \pi_{1}(G)\right)$. In fact, it is not difficult to show that

$$
\hat{\Phi}\left(C, A_{h}^{g v}\right)=\hat{\Phi}\left(C, A_{h}\right),
$$

where $g_{U}=[U]$ is the set of all those gauge transformations that are related to $U$. The set of all $\left\{g_{U}\right\}$ constitute the abelian group $H^{1}$. Evidently (4.4) indicates that $\hat{\Phi}$ is gauge invariant. However, this time we will have different Wilson loops that are labelled by the elements of $\operatorname{HOM}\left(\pi_{1}(M) \rightarrow G\right)$ as:

$$
\hat{\Phi}\left(C, R, A_{h}\right) \equiv \hat{\Phi}_{h}\left(C, R, A_{h}\right) .
$$

Consequently, the generating functional (1.9) is gauge invariant but classified according to $\pi_{1}(M)$. In fact, there are $h$ of them; $h=\operatorname{dim} \operatorname{HOM}\left(\pi_{1}(M) \rightarrow G\right)$ and we label them by $W_{h}(M, C, R)$. Our analysis in Sect. 2 can be repeated to work out the loop equation of $W_{h}$. The only difference this time is that rather having one Eq. (2.7) [or (2.10)] we have now $h$ of them. Hence we will have $h$ solutions. For $h \neq h^{\prime}$ the solutions $W_{h}$ and $W_{h^{\prime}}$ lead to two different quantum field theories. The next question is how to calculate $Z_{h}$ for non-simply connected manifolds. The first significant modification is that the spinors $\psi_{\lambda}^{\alpha}$ will now couple to the classical fields $A_{h}$. In other words the covariant derivative in the Fermionic action must be covariantized with respect to the gauge fields $A_{h}$. However, as soon as we turn on the coupling of the gauge fields $A_{h}$ to the spinors the partition function $Z$ loses gauge invariance under the large gauge transformation $g_{u}=[U]$ of $H^{1}\left(M, \pi_{1}(G)\right.$ ). In fact, the gauge fields $A_{h}$ get lifted up to another class of gauge fields $\left(A_{h}\right)_{g}$ which are classified by the $H^{1}$ as well as $\operatorname{HOM}\left(\pi_{1}(M) \rightarrow G\right)$. Of course, this situation does not occur in the coupling to the Chern-Simons gauge theory. In other words $H^{1}$ comes into the game only when there is coupling to spinors $[11,12]$. Consequently, the Fermionic partition functions $\left(Z_{h}\right)_{g}$ are $H^{1}$ gauge dependent and they are classified by the first cohomology group of $M_{3}$, their expressions are given as in (3.24) and (3.25), however, with the modification that the covariant derivative $D_{e}$ is covariantized with respect to the gauge fields $\left(A_{h}\right)_{g}$. On physical grounds, however, we will be interested in gauge invariant quantum field theory and therefore, we will be concerned in constructing $Z_{h}$ that is gauge invariant. Such a situation has been tackled before within the context of quantized spinor fields on non-simply connected manifolds [12]. In fact, there are two good candidate solutions: the first one is taking the sum over all $\left(Z_{h}\right)_{g}$ :

$$
Z_{h}[S]=\sum_{g \in H^{\prime}} \chi(g) \cdot\left(Z_{h}\right)_{g}[S],
$$

where the weights $\chi(g)$ are some complex numbers that are appropriately chosen and they label the different gauge invariant vacuum states that are associated with 
the Chern-Simons gauge field $\left(A_{h}\right)_{g}$. In fact, they are the characters of the abelian group $H^{*}$ dual to $H^{1}$ and satisfy the following properties:

$$
\begin{gathered}
\chi(1)=1, \\
\chi(g) \cdot \chi\left(g^{\prime}\right)=\chi\left(g g^{\prime}\right),
\end{gathered}
$$

and using the fact that $H^{1}$ is abelian (i.e. $\left.g^{2}=1, g \in H^{1}\right)$ we deduce from $(4.7 \mathrm{a}, \mathrm{b})$ that:

$$
(\chi(g))^{2}=1 .
$$

Another candidate is to take the product of $\left(Z_{h}\right)_{g}$ :

$$
Z_{h}(M, L)=\prod_{g \in H^{\prime}}\left(Z_{h}\right)_{g}(M, L) .
$$

In fact, Eqs. (4.6) and (4.8) can be combined into one single candidate:

$$
\begin{gathered}
Z_{h}\left(M, L, g_{1}, \ldots, g_{s}\right)=\sum_{g \in H^{\prime}} \chi(g) \cdot\left(Z_{h}\right)_{g g} \ldots\left(Z_{h}\right)_{g g}, \\
g_{i} \neq g_{j}, \quad s=1 \ldots \operatorname{dim} H^{1}, \quad i \neq j .
\end{gathered}
$$

Equation (4.9) will be our solution for the quantum non-critical Fermionic string theory for non-simply connected 3-d manifolds. Using Eqs. (4.7) we can prove again the identities (A)-(B)-(C), however, the situation is now more delicate and the identities have to be properly stated, in particular, the identity (B). This is because it involves the gluing of two non-simply connected 3-d manifolds which have different non-trivial homotopy classes associated with the fundamental homotopy group. The question we are interested in is given $\pi_{1}\left(M_{1}\right)$ and $\pi_{1}\left(M_{2}\right)$ what can we learn about $\pi_{1}\left(M_{1} \cup M_{2}\right)$ assuming that $M_{1}$ and $M_{2}$ have boundaries $\Sigma_{1}$ and $\Sigma_{2}$ that have been identified and that these boundaries are compact Riemann surfaces of genus $g$. This is a purely algebraic topology problem and the best answer one can provide is contained in the following commutative diagram [14]:

$$
\begin{array}{ccc}
\pi_{1}(\Sigma) & \rightarrow & \pi_{1}\left(M_{1}\right) \\
\downarrow & \downarrow \\
\pi_{1}\left(M_{2}\right) & \rightarrow & \pi_{1}\left(M_{1} \cup M_{2}\right)
\end{array}
$$

which indicates that when pulled back to $\pi_{1}(\Sigma)$, the representation of $\pi_{1}\left(M_{1} \cup M_{2}\right)$ is the same as a pair of representations of $\pi_{1}\left(M_{1}\right)$ and $\pi_{1}\left(M_{2}\right)$. This is the Seifert-Van Kampen theorem. If the compact 2-manifold is simply connected $\left(S^{2}\right)$ then $\pi_{1}\left(M_{1} \cup M_{2}\right)$ is the free product of the groups $\pi_{1}\left(M_{1}\right)$ and $\pi_{1}\left(M_{2}\right)$ with respect to the homomorphisms $f_{1}: \pi_{1}\left(M_{1}\right) \rightarrow \pi_{1}(M)$ and $f_{2}: \pi_{1}\left(M_{2}\right) \rightarrow \pi_{1}(M)$, where $M=M_{1} \cup M_{2}$. Consequently, the identity (B) is stated as:

$$
W_{h^{\prime \prime}}\left(M_{1} \cup M_{2}, L_{1}+L_{2}\right) \cdot W_{k}(\Sigma, C)=W_{h}\left(M_{1}, L_{1}\right) \cdot W_{h^{\prime}}\left(M_{2}, L_{2}\right),
$$

where $\left[h^{\prime \prime}\right]$ and $[k]$ are specified by (4.10). For a simply connected $\Sigma,[k]=0$ and $\left[h^{\prime \prime}\right]=[h] *\left[h^{\prime}\right][15]$. Finally, we would like to know whether or not the solutions for the $W_{h}$ loop equations are the quantum non-critical Fermi string theories $Z_{h}$ (4.9). The solution (3.31) as it stands breaks down because on a general non-simply connected manifold it is not possible to find a surface $S$ whose boundary is the loop 
$C$. For example, the three torus, here the loop which winds around $T^{3}$ is not the boundary of any surface $S$. However, there is a class of non-simply connected manifolds in which the surface $S$ exits and such that any loop $C$ can be the boundary of some surface $S$ embedded in $M_{3}$, these manifolds are known as the homology 3-spheres. These are precisely the kind of manifolds that Mathematicians are interested in!. The reason is the following: the representations $[h]$ defining $H^{1}(M, G) \simeq \operatorname{HOM}\left(\pi_{1}(M) \rightarrow G\right)$ contain in general reducible representations for a non-abelian gauge group $G$. For example, consider the case where $G=S U(2)$, the reducible representations $\left[h_{u}\right]=[U]$ are classified by all those gauge transformations $U$ (1.3) for which

$$
A_{h}^{U}=A_{h},
$$

where $A^{U}$ is given by (1.3). The connections $A_{h}(4.12)$ are called reducible. Such representations correspond to singular points in the moduli space $H^{1}(M, G)$ and therefore, one would like to avoid them by imposing a restriction on $H^{1}$ i.e. working on a subspace of the moduli space in which all the representations $[h]$ are irreducible. If $G=S U(2)$ these reducible representations correspond to elements of $H^{1}\left(M, S^{1}\right)$. Hence the required restriction is to set $H_{1}(M)$ to be equal to zero. Manifolds with this property are known as homology 3-spheres. For $G=S U(N)$, or other non-abelian groups the situation is more complicated, in the sense of identifying the reducible representations. This is a mathematically open problem. Therefore, on homology 3-spheres the solution of the regularized loop equation is again a non-critical Fermionic string theory:

$$
W_{h}\left(M_{3}, C, R\right)=\hat{\Phi}_{h}(C, R) \cdot Z_{h}^{-1}\left(M_{3}\right) \cdot \sum_{\substack{S \in M^{3} \\ \partial S=C}} \cdot Z_{h}\left(M_{3}, S, R\right) e^{\alpha l_{C}},
$$

where $Z$ is given in Eq. (4.9).

\section{Conclusion}

In this paper we presented a new approach for quantizing Chern-Simons gauge theory in $d=3$ based on the geometry of quantum loops. We showed that the quantum loop equation is linear for unlinked loops on $S^{3}$ and as a consequence we proved that the theory is exactly soluble in loop space. The partition function of the Wilson loop is reparametrization invariant. This, together with the linearity of the loop equation allowed us to find explicit solutions among which is a noncritical Fermi string theory. Consequently, we rederived some important knot identities [6]. In particular, we obtained the value of the partition function for an unknotted Wilson line on $S^{3}$ as the characters of $S U(2)$. Finally, we presented the generalization of our constructions to 3-d non-simply connected manifolds which are homology 3-spheres. We showed that the solutions of the loop equations are Fermionic string theories classified by the homotopy classes of $\pi_{1}(M)$ and $H^{1}\left(M, \pi_{1}(G)\right)$.

Acknowledgements. I would like to express my sincere thanks to C. J. Isham for very constructive discussions on the topology of three-dimensional manifolds, N. Manojlovic and D. Olive for fruitful conversations and explaining to me the relevance of characters in the above constructions, and E. Witten for communicating his work which has motivated this paper. A. Neveu, R. Wilkinson, J. Soto, and A. Achucarro for very useful discussions. 


\section{References}

1. Wilson, K.: Phys. Rev. D 10, 2445 (1974)

2. Kogut, J., Susskind, L.: Phys. Rev. D 11, 395 (1975)

3. Polyakov, A.M.: Nucl. Phys. B 164, 171 (1979); Phys. Lett. 82 B, 247 (1979)

4. Migdal, A.A.: Ann. Phys. 126, 279 (1980); Makeenko, Y.M., Migdal, A.A.: Nucl. Phys. B 188, 269 (1981)

5. Migdal, A.A.: Nucl. Phys. B 189, 253 (1981)

6. Witten, E.: IASSNS preprints, Sept. 1988

7. Awada, A.: Imperial College preprint. Nov. 1988

8. Tze, C.: Int. Mod. Phys. A 3, 1959 (1988)

9. Polyakov, A.M.: Mod. Phys. Lett. A 3, 325 (1988)

10. Casher, A., Foerster, D., Windey, P.: Nucl. Phys. B 251, 29 (1985)

11. Isham, C.J.: In: Essays in honour of Wolfgang Yourgrau. van der Merwe, A. (ed.). London: Plenum Press 1981

12. Isham, C.J., Kunstatter: Phys. Lett. 102 B, 417 (1981); Isham, C.J., Avis, A.: Nucl. Phys. B 156, 441 (1979)

13. Hamermesh, M.: Group theory. Reading, MA: Addison-Wesley 1962

14. Atiyah, M. F.: In: The mathematical Heritage of Hermann Weyl. Proc. Symp. Pure Math. 48. Wells, R. (ed.), Providence, RI: American Mathematical Society 1988

15. Hempel, J.: 3-Manifolds, Ann. Math. Stud., vol. 86. Princeton, NJ: Princeton University Press 1976

16. Deser, S., Jackiw, R., Templeton, S.: Phys. Rev. Lett. 48, 975 (1983); Ann. Phys. NY 140, 372 (1984); Zuckerman, G.: In: The Proceedings of the 1986 San Diego Summer Workshop, Yau, S. T. (ed.)

17. Schwarz, A.: Lett. Math. Phys. 2, 247 (1978)

18. Awada, M. A.: Imperial college preprint TP/88-89/13

19. Kauffman, L.H.: "On Knots," Princeton, NJ: Princeton Univ. Press 1987; Jones, V.F.R.: Bull. Am. Math. Soc. 12, 103 (1985); Ann. Math. 126, 335 (1987); Lickorish, W.B.R., Millett, K.C.: Topology 26, 107 (1987)

Communicated by L. Alvarez-Gaumé

Received January 13, 1989; in revised form August 10, 1989 
